\title{
Knockout of PARG110 confers resistance to cGMP-induced toxicity in mammalian photoreceptors
}

\author{
A Sahaboglu ${ }^{*, 1}$, N Tanimoto ${ }^{2}$, S Bolz ${ }^{1}$, MG Garrido ${ }^{2}$, M Ueffing ${ }^{1}$, MW Seeliger ${ }^{2}$, H Löwenheim ${ }^{3}$, P Ekström ${ }^{4}$ and F Paquet-Durand ${ }^{*, 1}$
}

Hereditary retinal degeneration (RD) relates to a heterogeneous group of blinding human diseases in which the light sensitive neurons of the retina, the photoreceptors, die. RD is currently untreatable and the underlying cellular mechanisms remain poorly understood. However, the activity of the enzyme poly-ADP-ribose polymerase-1 (PARP1) and excessive generation of poly-ADPribose (PAR) polymers in photoreceptor nuclei have been shown to be causally involved in RD. The activity of PARP1 is to a large extent governed by its functional antagonist, poly-ADP-glycohydrolase (PARG), which thus also may have a role in RD. To investigate this, we analyzed PARG expression in the retina of wild-type ( $w t)$ mice and in the rd1 mouse model for human RD, and detected increased PARG protein in a subset of degenerating rd1 photoreceptors. Knockout (KO) animals lacking the $110 \mathrm{kDa}$ nuclear PARG isoform were furthermore analyzed, and their retinal morphology and function were indistinguishable from wildtype animals. Organotypic wt retinal explants can be experimentally treated to induce rd1-like photoreceptor death, but PARG110 KO retinal explants were unexpectedly highly resistant to such treatment. The resistance was associated with decreased PAR accumulation and low PARP activity, indicating that PARG110 may positively regulate PARP1, an event that therefore is absent in PARG110 KO tissue. Our study demonstrates a causal involvement of PARG110 in the process of photoreceptor degeneration. Contrasting its anticipated role as a functional antagonist, absence of PARG110 correlated with low PARP activity, suggesting that PARG110 and PARP1 act in a positive feedback loop, which is especially active under pathologic conditions. This in turn highlights both PARG110 and PARP1 as potential targets for neuroprotective treatments for RD.

Cell Death and Disease (2014) 5, e1234; doi:10.1038/cddis.2014.208; published online 22 May 2014

Subject Category: Neuroscience

Hereditary neurodegenerative diseases of the retina, such as retinitis pigmentosa, lead to photoreceptor cell death and blindness. These diseases affect approximately 1 in 3000 people and remain to this day without treatment, ${ }^{1}$ which to a considerable degree is a consequence of the insufficient understanding of the underlying cellular disease mechanisms. Nevertheless, recent studies on animal models for hereditary photoreceptor degenerations, such as the rd1 mouse, have advanced the knowledge on the pathological processes. ${ }^{2,3}$ For example, in the rd1 retina, the activation of poly-ADPribose-polymerase-1 (PARP1), with a following excessive accumulation of poly-ADP-ribose (PAR) in the nuclei of dying photoreceptors, ${ }^{4,5}$ has been linked to the retinal degeneration (RD) characteristics of this model. Moreover, similar observations on PARP hyperactivity and PAR accumulation have been made in several other relevant animal models. ${ }^{6}$

PARP1 is likely the most abundant nuclear protein in an enzyme family coming from at least 18 different genes ${ }^{7}$ and that mediates the addition of PAR entities to substrate proteins in a process, which can be referred to as PARylation. PARylation represents a post-translational protein modification that is important for nuclear chromatin structure and transcriptional activity but that also governs the functions of many other cellular proteins and processes. ${ }^{8}$ Remarkably, the PARP1 enzyme PARylates its own automodification domain to inhibit and limit the PARP activity in what appears to be an autoregulatory feedback loop. ${ }^{9}$

The rd1 mouse is a well-studied mouse model for RD and suffers from a human homologous mutation in the gene encoding for the beta subunit of rod photoreceptor cGMP phosphodiesterase-6 (PDE6). ${ }^{10}$ The PDE6 dysfunction leads to a strong rise in cGMP and subsequent rd1-photoreceptor death. ${ }^{5}$ Interestingly, pharmacological inhibition of PDE6 by the selective inhibitor zaprinast ${ }^{11}$ will emulate rd1-like degeneration on non-rd1-retinas, ${ }^{12,13}$ such that otherwise healthy retinas treated with zaprinast show large numbers of dying photoreceptors. ${ }^{5}$ However, retinas obtained from PARP1 knockout (KO) animals are resistant to zaprinast treatment, reinforcing the notion that PARP1 drives-at least in part-photoreceptor degeneration.

Another key player in the PAR metabolism is poly-ADPribose-glycohydrolase (PARG), which in turn removes PAR from target proteins. ${ }^{9}$ In this context, PARG seems to be a major regulator for PARP1 as it removes inhibitory PAR

\footnotetext{
${ }^{1}$ Division of Experimental Ophthalmology, Institute for Ophthalmic Research, University of Tübingen, Tubingen, Germany; ${ }^{2}$ Division of Ocular Neurodegeneration, Institute for Ophthalmic Research, Tuebingen, Germany; ${ }^{3}$ Department of Otolaryngology, Tuebingen Hearing Research Centre, Tuebingen, Germany and ${ }^{4}$ Division of Ophthalmology, Department of Clinical Sciences, University of Lund, Lund, Sweden

*Corresponding author: A Sahaboglu or F Paquet-Durand, Division of Experimental Ophthalmology, Institute for Ophthalmic Research, University of Tübingen, Röntgenweg 11, Tübingen 72076, Germany. Tel: +49 0707129 84781; Fax: +49 707129 5777; E-mail: aysesahaboglu@ hotmail.com (AS) or Tel: +49 7071 29 87430; Fax: +49 707129 5777; E-mail: francois.paquet-durand@ klinikum.uni-tuebingen.de (FP-D)

Keywords: TUNEL; PARP; necrosis; retina; CGMP

Abbreviations: HDAC, histone deacetylase; IF, immunofluroscence; KO, knockout; ONL, outer nuclear layer; PDE6, phosphodiesterase-6; PARP, poly-ADP-ribosepolymerase; PARG, poly-ADP-glycohydrolase; RD, retinal degeneration; TUNEL, terminal deoxynucleotidyl transferase dUTP nick end labeling; wt, wild-type Received 06.2.14; revised 20.3.14; accepted 09.4.14; Edited by A Verkhratsky
} 
polymers from the PARP1 automodification domain, ${ }^{14,15}$ thus allowing reactivation of PARP1. In mammals, there is a single Parg gene, highly conserved among mammals ${ }^{16}$ and giving rise to at least five PARG isoforms with different subcellular localizations and molecular weights. ${ }^{8,17}$ Among these, the $110 \mathrm{kDa}$ isoform (PARG110) is the only one localizing to the nucleus, ${ }^{18}$ which makes it an obvious candidate for a putative interaction with the hyperactivated nuclear PARP as seen in degenerating photoreceptors. This motivated us to study the connection of PARG, and particularly PARG110, with RD.

In the present work, we show that PARG is expressed in all retinal layers, and that its expression increases in individual degenerating $r d 1$ photoreceptors. Although $\mathrm{KO}$ of the PARG110 isoform $^{19}$ does not seem to affect the retinal morphology and function as such, the photoreceptor cell death response to pharmacological PDE6 blockage is strongly reduced in $\mathrm{KO}$ retina. This suggests a mechanistical involvement of PARG110 in photoreceptor cell death, possibly via (re)activation of the detrimental PARP1.

\section{Results}

PARG expression is increased in degenerating rd1 photoreceptors. Because of the nuclear localization of PARP1 activity and PAR accumulation observed during photoreceptor cell death, ${ }^{4,5}$ we were particularly interested in the nuclear PARG110 isoform in the context of RD. To address the potential role for PARG110 in RD, we first assessed its retinal expression using immunofluorescence (IF) with a PARG antibody that detects both the 110 and $56 \mathrm{kDa}$ isoforms. The specificity of the antibody was confirmed using tissue from animals in which the PARG110 isoform had been genetically deleted. ${ }^{19}$ The IF experiments indicated PARG110 expression in all retinal cells in the wild-type (wt) situation, albeit with a particularly strong expression in the nerve fiber layer (NFL) and in the perinuclear areas of cells that morphologically resembled amacrine cells and horizontal cells (Figure 1a). The latter was confirmed using co-staining with an antibody against calbindin (Figures $1 \mathrm{~b}$ and $\mathrm{c}$ ), a prototypic marker for horizontal cells. ${ }^{20}$ In PARG110 KO, overall PARG staining was reduced, especially so in the horizontal cells, but not in the NFL (Figures 1d-f). This suggests that the PARG antibody indeed recognizes PARG110 and that the horizontal cells rely extensively on this PARG isoform. By contrast, other retinal structures, such as the NFL, may preferentially express other PARG isoforms. We then studied PARG expression in a situation of degeneration: Although in wt photoreceptors, PARG expression was very low (Figure 1a), in rd1 outer nuclear layer (ONL) there was a strong PARG upregulation in the perinuclear regions of many photoreceptors (Figure 1g). At the same time, the localization to horizontal and amacrine cells appeared to be unchanged (Figures $1 \mathrm{~h}$ and $\mathrm{i}$ ). The latter result indicated a possible involvement of PARG110/PARG56 in RD, with the perinuclear localization pointing towards PARG110.

PARG110 KO retina is morphologically and functionally normal. To study the importance of nuclear PARG110, we assessed retinal morphology and function in PARG110 KO animals using both in vivo and ex vivo techniques. A gross morphologic comparison of PARG110 $\mathrm{KO}$ and $w t$ retinae at P30 did not reveal major differences in terms of retinal thickness and layering, neither in ex vivo histology (Figures $2 a$ and b) nor in in vivo optical coherence tomography (OCT) imaging (Figures $2 \mathrm{c}$ and d). A detailed histological analysis of photoreceptor rows in early postnatal retina at $\mathrm{P} 11$ for $w t$ (11.9 \pm 0.9 S.E.M., $n=3$ and PARG110 KO retina $(9.8 \pm 0.5$ S.E.M., $n=3$ ) showed comparable numbers (Figure 2e; $P=0.111)$. A similar picture for the photoreceptor row counts was seen at P30 (wt: $13.1 \pm 0.1$ S.E.M., $n=3$; PARG110 KO: $13.0 \pm 0.8$ S.E.M., $n=3, P=0.912)$. The measurement of ONL thickness also showed no major differences between $w t$ $(64.8 \mu \mathrm{M} \pm 0.5 \quad$ S.E.M., $\quad n=3)$ and PARG110 KO (56.4 $\mu \mathrm{M} \pm 3.2$ S.E.M., $n=3, P=0.063$ ) at $\mathrm{P} 11$ and at $\mathrm{P} 30$ (wt: $62.2 \mu \mathrm{M} \pm 0.4$ S.E.M., $n=3$; PARG110 KO: $61.3 \mu \mathrm{M} \pm 3$ S.E.M., $n=3 ; P=0.797$, Figure 2e). As histone PARylation may affect chromatin condensation, nuclear structure, and possibly cell density in the ONL, ${ }^{21}$ we also assessed the size of photoreceptor nuclei at PN30 and found no significant differences between the two genotypes (wt: $13.9 \pm 0.2$ S.E.M., $n=3$; PARG110 KO: $13.7 \pm 0.3$ S.E.M., $n=3$, $P=0.523)$.

Single-flash electroretinograms (ERGs) elicited by full-field light stimulation were comparable between wt and PARG110 $\mathrm{KO}$ mice under both scotopic and photopic conditions at all intensities examined (Figures $2 f$ and $g$ ), indicating no aberrations of rod and cone photoreceptor function at P30.

No evidence for abnormal cell death in PARG110 KO retina. To exclude the possibility of a slow, low-level degeneration in PARG110 KO that was not detectable with conventional histology, we studied a number of cell death markers typically associated with photoreceptor degeneration. For comparative purposes, these markers were also assessed in PARP1 KO retina. Localization of PDE6B protein as a marker for the integrity of photoreceptor outer segments failed to show any abnormalities (Supplementary Figure 1). In line with this, there was no evidence for cGMP accumulation in wt, PARG110 KO, or PARP1 KO retina (Supplementary Figure 1). Likewise, the terminal deoxynucleotidyl transferase dUTP nick end labeling (TUNEL) assay showed a very low percentage of positive cells for $w t$ $(0.06 \% \pm 0.01$ S.E.M., $n=3)$, PARP1 KO $(0.04 \% \pm 0.02$ S.E.M., $n=3)$, and PARG110 KO $(0.03 \% \pm 0.03$ S.E.M., $n=3$ ) retina, with no statistical differences between either of these genotypes.

The PARP activity assay, which acts as a cell death marker in $r d 1$ retina, showed very low levels of PARP activation in wt $(0.02 \% \pm 0.01$ S.E.M., $n=3)$, and no PARP activation in either PARP1 KO $(0.00 \% \pm 0.00$ S.E.M., $n=3)$ or PARG110 KO $(0.00 \% \pm 0.00$ S.E.M., $n=3)$ retina. Although numerically small, a one-way analysis of variance (ANOVA) analysis showed that there were statistically significant differences in the wt and PARG110 KO and PARP1 KO data $(P<0.05$; Supplementary Figure 1). A subsequent Student's $t$-test gave at hand that the difference was between wt and PARG1 KO $(P=0.017$, which also after Bonferroni correction will be regarded as statistically significant).

The accumulation of PARylated proteins indirectly indicates PARP activity. ${ }^{4}$ At P11, PAR immunostaining showed very 

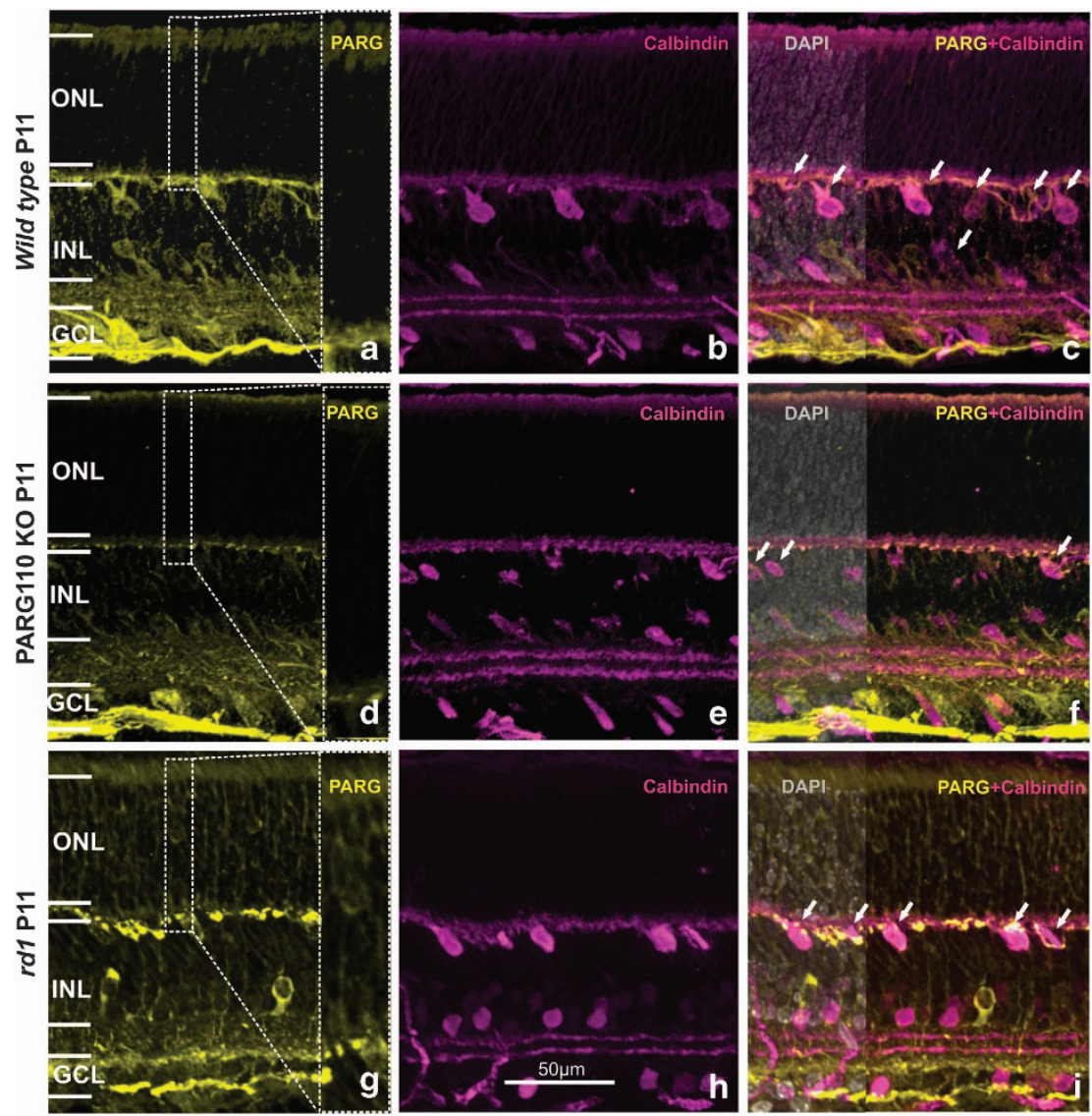

Figure 1 Retinal PARG expression in different genotypes: In wt retina, PARG expression was particularly evident in the NFL and in the perinuclear parts of a subpopulation of amacrine cells and horizontal cells (white arrows), as assessed by co-staining with calbindin (a-c). In PARG110 KO, PARG expression in perinuclear areas of amacrine and horizontal cells (white arrows) was strongly reduced, while PARG levels in the synaptic layers and the NFL appeared to be unaffected (d-f). In rd1 retina, the perinuclear areas of many photoreceptors displayed distinct PARG expression ( $\mathbf{g}-\mathbf{i})$, in contrast to the $w t$ situation (white arrows indicate horizontal cells). The images shown are representative for observations on at least three different specimens for each genotype

few positive photoreceptors in wt and PARG110 KO animals (wt: $0.03 \% \pm 0.01$ S.E.M., $n=3$; PARG110 KO: $0.02 \% \pm 0.01$, $n=6)$, while in PARP1 $\mathrm{KO}$ retina no positive cells were seen $(0.00 \% \pm 0.00$ S.E.M., $n=6$; Supplementary Figure 1). A oneway ANOVA followed by Student's $t$-test indicated a statistically significant difference between the PAR values of wt and PARP1 KO.

rd1 RD is causally related to increased histone deacetylase (HDAC) activity. ${ }^{22}$ In the present work, the HDAC activity assay identified only few positive cells ( $w t$. $0.03 \% \pm 0.01$ S.E.M., $n=3$; PARP1 KO: $0.01 \% \pm 0.01$ S.E.M., $n=3$; PARG110 KO: $0.03 \% \pm 0.01$ S.E.M., $n=3, P=0.915$ ), without any significant differences between either of these genotypes.

The calcium-dependent protease calpain has repeatedly been implicated in photoreceptor cell death in a variety of RD models ${ }^{6,19}$ and would therefore also qualify as a cell death marker here. The activity assay for calpain detected no or hardly any positive cells for wt, PARP1 KO (wt: 0.01\% \pm 0.00 S.E.M., $n=3$; PARP1 KO: $0.01 \% \pm 0.01, n=3)$, and PARG110 KO $(0.00 \% \pm 0.00$ S.E.M., $n=3)$.

In summary, in vivo and ex vivo examinations found no adverse effects of PARG110 KO on either retinal function or morphology. These results in turn suggested that neuroprotective strategies aimed at inhibiting PARG110 would not negatively affect photoreceptor retinal cell survival. The data further suggested that both the lack of PARG110 and PARP1 will affect the detectable PARP activity or PAR accumulation in photoreceptors.

Absence of PARG110 confers resistance to RD. In order to further delineate the role of PARG110 during RD, we treated organotypic PARG110 KO retinal explants with the PDE6 specific inhibitor zaprinast. ${ }^{11,13}$ This treatment raises photoreceptor cGMP levels and pharmacologically induces photoreceptor degeneration in ways very similar to what is observed in the Pde6b mutant $r d 1$ retina. $^{12,23}$ Although untreated retina was negative for CGMP, a strong, zaprinastinduced rise in photoreceptor cGMP levels was evident in both $w t$ and PARG110 KO retina (Figures $3 a-d)$.

Detection of dying cells with the TUNEL assay identified few positive ONL cells in untreated wt retinal explants (Figures $3 \mathrm{i}-\mathrm{I}$ ) (0.74\% \pm 0.27 S.E.M., $n=6)$, and a strongly increased number for the zaprinast-treated $w t(3.91 \% \pm 0.55$ S.E.M., $n=6, \quad P=0.0004)$. Untreated PARG110 (1.37\% 0.14 S.E.M., $n=6)$ and zaprinast-treated PARG110 retinal explants $(1.16 \% \pm 0.20$ S.E.M., $n=6)$ both displayed low numbers of TUNEL positive cells in their ONL. Importantly, a comparison between zaprinast-treated $w t$ and PARG110 KO 
wt

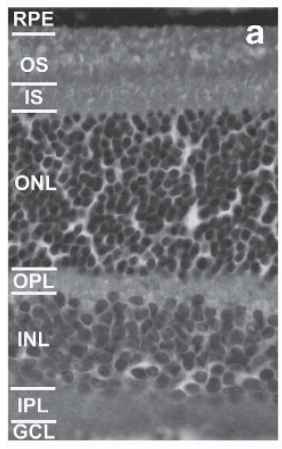

e
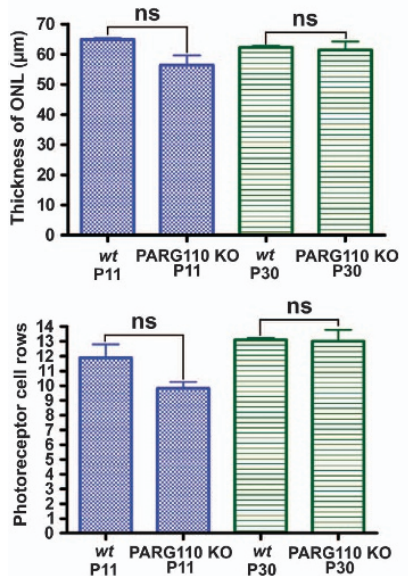

PARG110 KO

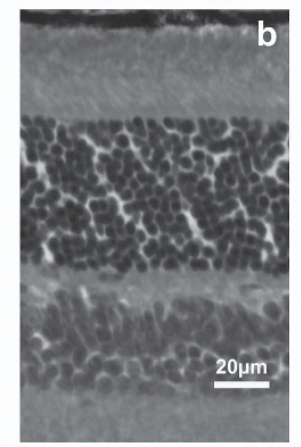

f

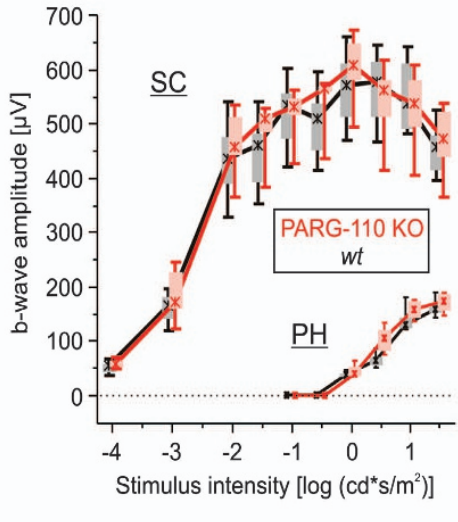

wt

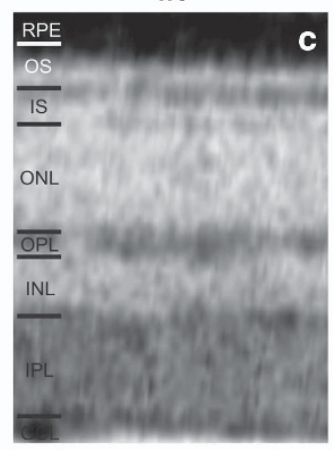

PARG110 KO

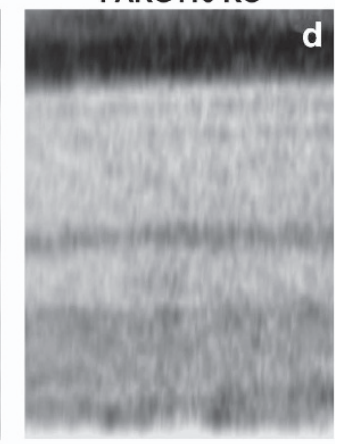

g
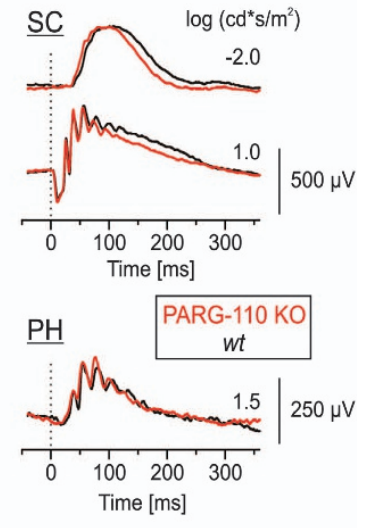

Figure 2 Retinal morphology and function is normal in PARG110 knockout animals. At P30, haematoxylin/eosin staining revealed normal morphology and layering for PARG110 KO retina, when compared to age-matched $w t$ (a and $\mathbf{b}$ ). OCT in vivo imaging confirmed the histological results (c and $\mathbf{d})$. The quantification of photoreceptor rows in the outer nuclear layer (ONL) and ONL thickness showed no significant differences between wt and PARG110 KO retina at day 11 (P11) and day 30 (P30) after birth (e). In vivo functional analysis with ERG did not detect any differences between wt (black) and PARG110 KO (red) mice under dark-adapted (scotopic; SC) and light-adapted (photopic; PH) conditions ( $\mathbf{f}$ and $\mathbf{g}$ ). Statistical evaluation of b-wave amplitudes (box-and-whisker plot, indicating 5, 25, 50, 75, 95 percentiles of the data) and comparison of representative ERG traces are shown in (f) and (g), respectively. Error bars represent S.E.M. GCL, ganglion nuclear layer; INL, inner nuclear layer; IPL, inner plexiform layer; IS, inner segment; ns, not significant; ONL, outer nuclear layer; OPL, outer plexiform layer; OS, outer segment; RPE, retinal pigment epithelium

revealed a highly significant difference $(P<0.001$; Figure $3 r)$, suggesting that lack of PARG110 expression and/or activity was neuroprotective.

Likewise, the numbers of cells showing high calpain activity (Supplementary Figure 2d-f) were low in untreated and zaprinast-treated PARG110 KO retina (untreated PARG110 KO: $\quad 0.24 \% \pm 0.04 ; \quad$ zaprinast-treated PARG110 KO: $0.12 \% \pm 0.01 ; n=3 ; P=0.055)$.

Photoreceptor cell death in $r d 1$ retina is non-apoptotic and negative for typical apoptotic markers such as caspase-3 activity $^{2}$ (and unpublished data). Nevertheless, to exclude the possibility that the absence of PARG110 or the treatment with zaprinast would shift the balance of cell death mechanisms towards classical, caspase-dependent apoptosis, we also tested for the activation of caspase-3. Immunostaining for activated caspase-3 (Supplementary Figure $2 \mathrm{~g}-\mathrm{i}$ ) resulted in only a small number of positive cells, both for untreated $(0.12 \% \pm 0.04$ S.E.M.) and zaprinast-treated PARG110 KO retinae $(0.03 \% \pm 0.01$ S.E.M., $n=3, P=0.128)$.

Taken together, all of these results suggested a resistance to zaprinast-induced retinal photoreceptor cell death in PARG110 KO animals.
Absence of PARG110 disrupts the PARP activation. The retinal explants experiments also revealed important aspects on PARP activation in PARG110 KO retinae. PAR presence, as seen by immunohistochemistry, (Figures $3 i-I$ ), is an index of PARP activity, and demonstrated a strong and significant $(P<0.01)$ increase in positive ONL cells in zaprinast-treated $w t$ specimens but not in zaprinast-treated PARG110 KO retina (untreated wt. $0.13 \% \pm 0.03$ S.E.M., $n=3-4$; zaprinast-treated $w t$. $0.69 \% \pm 0.07$ S.E.M., $n=3-4 ; P=0.002$ : untreated PARG110 KO: $0.12 \% \pm 0.03$ S.E.M., $n=3-4$; zaprinast-treated PARG110 KO: $0.16 \% \pm 0.06$ S.E.M., $n=3-4 ; P=0.008$, Figure 3s). While this finding confirmed the close correlation between cell death and PAR accumulation, it was somewhat surprising as PAR accumulation is an indication of PARP activity. Decreased expression of the hydrolyzing enzyme PARG110 would hence be expected to lead to an increase in PAR accumulation.

To further address this issue, we also analyzed PARP activity in untreated and zaprinast-treated PARG110 KO retina (Supplementary Figure $2 \mathrm{a}-\mathrm{c}$ ) and found PARP activity to be essentially the same in both types (untreated PARG110 KO: $0.21 \% \pm 0.04$ S.E.M.; zaprinast-treated 

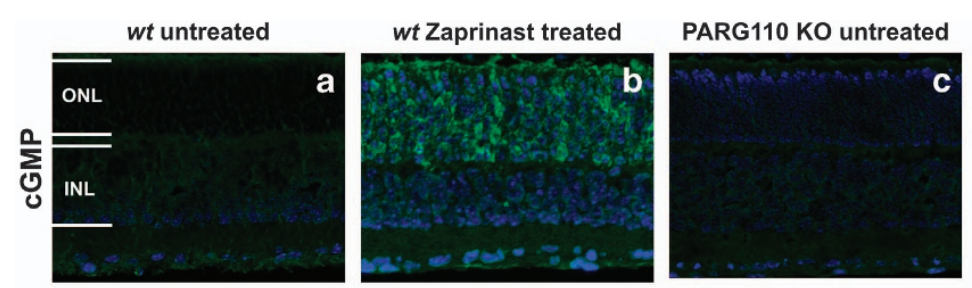

\section{PARG110 KO Zaprinast}
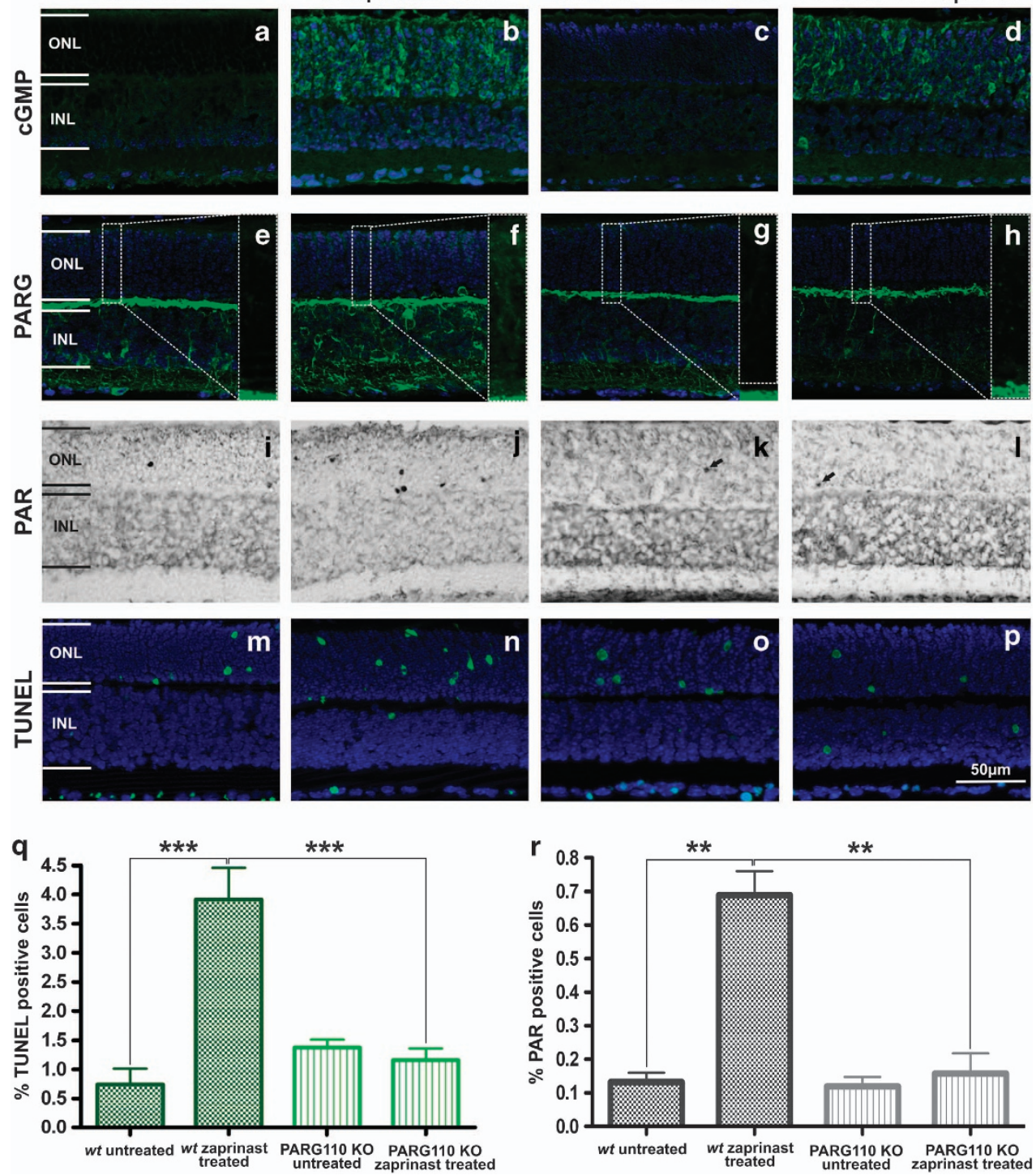

Figure 3 PARG110 KO photoreceptors are resistant to pharmacologically induced RD: organotypic retinal explants cultures derived from wt and PARG110 KO animals were treated with the PDE6 inhibitor zaprinast to induce an rd1-like retinal degeneration. Untreated wt and PARG110 KO explants exhibited minimal IF for cGMP, while zaprinast-treated specimens showed strongly increased ONL cGMP levels (a-d). PARG expression was seen only in the nerve fiber layer and in the perinuclear parts of a subpopulation of amacrine cells and horizontal cells for untreated wt, untreated PARG110 KO and zaprinast-treated PARG110 KO. However, in zaprinast-treated wt, an increased PARG expression was observed in the ONL and INL (e-h). PAR accumulation, a marker for PARP activity, was found in large amounts only in zaprinast-treated wt ONL (i-I), but rarely in untreated wt or in either treated or untreated PARG110 KO. The TUNEL assay for dying cells showed few positive cells in untreated wt but increased numbers in zaprinast-treated $w t(\mathbf{m}$ and $\mathbf{n})$. Zaprinast-treated and untreated PARG110 KO retinal explants showed equally low numbers of TUNEL positive cells ( $\mathbf{o}$ and $\mathbf{p})$. Bar graphs show the quantification of TUNEL- (q) or PAR- $(\mathbf{r})$ positive ONL cells. Error bars represent S.E.M. INL, inner nuclear layer; ONL, outer nuclear layer. Levels of significance: ${ }^{* \star} P<0.01,{ }^{* * *} P<0.001$

PARG110 KO: $0.26 \% \pm 0.141$ S.E.M.; $n=3 ; P=0.737$ ). This finding suggests that the PARG110 KO retina is lacking the ability to activate, or keep activated the PARP enzyme.

\section{Discussion}

The present study provides for the first time data showing that PARG is expressed in the retina and that PARG110 is causally involved in neurodegeneration of photoreceptors. Moreover, it offers novel information on the interplay of PARP and PARG during photoreceptor neurodegeneration.
PARG in the retina. Although PARG expression is thought to be ubiquitous, ${ }^{24}$ a retinal PARG expression has not been shown before. Here, we demonstrate that while probably expressed in all retinal cell types, the 110 and $56 \mathrm{kDa}$ isoforms in the normal, healthy retina appear to be particularly strongly expressed in the NFL, in horizontal cells, and cells that morphologically resembled amacrine cells. Moreover, when studied in the rd1 retina, where there is ongoing photoreceptor degeneration, an increased PARG expression in many of the photoreceptors was observed. Interestingly, loss of PARG110 expression did not seem to affect the normal retinal cell survival, nor retinal function and 
morphology, at least when it comes to the parameters investigated here. Together these observations suggest that while the retinal role of PARG in the normal, healthy situation is subtle, its presence and activity becomes more important when the system is provoked.

Crosstalk between PARG and PARP. Whereas PARP1 has been implicated in cell death in a variety of disease conditions, ${ }^{25}$ including in retinal neurodegeneration, ${ }^{5}$ data on PARG in this context is very scarce. Surprisingly, in our study, loss of PARG110 expression led to an apparent decrease in PARP activity, suggesting that PARG110 formed part in a feedback loop that positively regulated PARP. This possibility is compatible with existing information. High PARP1 activity results in autoPARylation of PARP1 in the enzyme's automodification domain, ${ }^{25,26}$ which would mean that once activated, PARP1 shuts itself off automatically and then requires PARG for reactivation. The specific activity of PARG is considerably higher than that of PARP, and as such, the in vivo half-life of PAR is only approximately $1 \mathrm{~min}$ in DNA-damaged cells. ${ }^{16}$ Even when taking these capacities into consideration, the very high PARP activity in degenerating photoreceptors ${ }^{4}$ is likely to require increased access to PARG in order to be maintained, which in turn could explain the upregulation of PARG110 in rd1 photoreceptors. In this scenario, the here observed protective effect of PARG110 KO would be a consequence of PARP activity being kept low because the PARP1 autoinhibition can no longer be lifted by PARG.

An unchecked PARP-PARG feedback loop will have a bearing on the cellular energy metabolism as it would lead to an excessive consumption of NAD + and indirectly, via the $\mathrm{NAD}+$ rescue pathway, to ATP depletion. ${ }^{27}$ While this view has been challenged to some extent recently, ${ }^{28}$ the net effect of an absent activation of the PARP-PARG loop, as in the zaprinast provoked PARG110 KO explants, can nevertheless be expected to be beneficial for cell survival because this absence would reduce the strain on energy metabolism. ATP levels are critical for cellular survival, not the least for maintaining $\mathrm{Ca}^{2+}$ homeostasis. ${ }^{29}$ Therefore, an improved $\mathrm{Ca}^{2+}$ control could explain why the activity of $\mathrm{Ca}^{2+}$ -dependent calpain-type proteases is rather low in zaprinast-treated PARP1 and PARG110 KO retina when compared with zaprinast-treated $w t$ and $r d 1$ retina.

PARG as a therapeutic target. Previous studies have shown the importance of PARP activity for RD in the rd1 mouse ${ }^{30}$ or in retinas with zaprinast-induced $r d 1$-like degeneration. ${ }^{5}$ Moreover, the S334ter and $\mathrm{P} 23 \mathrm{H}$ rat models for RD show PARP activation during photoreceptor degeneration, ${ }^{6}$ as do several other animal models (unpublished data). PARP activation thus appears to be a common denominator in photoreceptor degeneration. Together with the fact that a number of animal studies have highlighted PARP inhibition as a promising new form of treatment for neurodegenerative diseases such as stroke and neurotrauma, ${ }^{9}$ this has made it very attractive to suggest anti-PARP therapeutic strategies for the genetically heterogeneous hereditary RDs.

We now propose that also PARG inhibition should be introduced as a therapeutic idea in this field. Loss of PARG activity by isoform-specific KO and knockdown of the Parg gene shows protective effects against, for example, $\mathrm{H}_{2} \mathrm{O}_{2}-$ induced cell death, ${ }^{31}$ and while the area of pharmacological PARG inhibitors is still limited, ${ }^{16,30}$ a small molecule PARG inhibitor has been shown to be protective in ischemic neurodegeneration. ${ }^{6}$ Furthermore, the fact that all PARG isoforms are encoded by a single PARG gene and that the cellular abundance of PARG proteins is very low, with approximately 2000 molecules per cell, ${ }^{30}$ suggests PARG as an attractive pharmacological therapeutic target. Continued research using genetic Parg knockdown approaches and possible future PARG inhibitors is very likely to add to the suggestion of PARG interference as a novel treatment option for inherited eye diseases.

\section{Conclusion}

Although several previous reports have shown the importance of PARP activity for RD, this is the first study on the role of PARG for photoreceptor cell death. We demonstrate that PARG110 is expressed in the retina and that in degenerating photoreceptors PARP activity and PAR accumulation depends on the expression of PARG110. This suggests that PARG110 is of major importance for maintaining the activity of PARP, and in turn highlights PARG inhibition as a novel approach for the development of therapies for hereditary RD.

\section{Materials and Methods}

Experimental animals. PARG110 KO, PARP1 KO and wild-type (wt) animals were housed under standard white cyclic lighting and had free access to food and water. PARG110 KO animals were kindly provided by Zhao-Qi Wang, Leibniz Institute for Age Research, Fritz Lipmann Institute, Jena, Germany. The PARG110 KO mice were generated by the deletion of exons 2 and 3 of the Parg gene resulting in depletion specifically of the PARG110 protein. ${ }^{19}$ PARG110 KO mice are phenotypically normal, although they exhibit hypersensitivity to radiation, DNA alkylating agents, and chemotherapeutics. ${ }^{5,19,30}$ All procedures were performed in accordance with the ARVO statement for the use of animals in ophthalmic and visual research and were approved by the Tübingen University committee on animal protection (Einrichtung für Tierschutz, Tierärztlicher Dienst und Labortierkunde directed by Dr. Franz Iglauer). Because of the critical molecular changes apparent in degenerating rd1 retina at postnatal day $(\mathrm{P}) 11,{ }^{2}$ comparisons between PARG110 KO, PARP1 KO, wt, and rd1 retinae were carried out at $\mathrm{P} 11$, and in addition in adult retina at $\mathrm{P} 30$.

Electroretinography. Full-field ERGs were recorded binocularly as described previously. ${ }^{32,33}$ Before experiments, mice were dark-adapted overnight. Anesthesia was induced by subcutaneous injection of ketamine $(66.7 \mathrm{mg} / \mathrm{kg}$ body weight) and xylazine (11.7 mg/kg body weight), and the pupils were dilated with tropicamide eye drops. Single-flash ERG responses were obtained under darkadapted (scotopic) and light-adapted (photopic) conditions. Light adaptation was accomplished with a background illumination of $30 \mathrm{~cd} / \mathrm{m}^{2}$ starting $10 \mathrm{~min}$ before photopic recording session. Single white-flash stimuli ranged from -4 to $1.5 \mathrm{log}$ $\mathrm{cd}^{\star} \mathrm{s} / \mathrm{m}^{2}$ under dark-adapted and from -2 to $1.5 \mathrm{log} \mathrm{cd}^{*} \mathrm{~s} / \mathrm{m}^{2}$ under light-adapted conditions. Ten responses were averaged with interstimulus intervals of $5 \mathrm{~s}$ (for -4 to $-0.5 \log \mathrm{cd}^{*} \mathrm{~s} / \mathrm{m}^{2}$ ) or $17 \mathrm{~s}$ (for 0 to $1.5 \log \mathrm{cd}^{*} \mathrm{~s} / \mathrm{m}^{2}$ ). Mice were examined at P30 (PARG110 KO, $n=3$ (six eyes); $w t, n=4$ (eight eyes)).

OCT. In vivo retinal layering was assessed by means of OCT with the Spectralis HRA + OCT device from Heidelberg Engineering (Heidelberg, Germany) featuring a broadband superluminescent diode at $\lambda=870 \mathrm{~nm}$ as low coherent light source. ${ }^{34}$ Each two-dimensional B-Scan recorded with the equipment set to $30^{\circ}$ field of view, consists of $1536 \mathrm{~A}$-scans acquired at a speed of 40000 scans per second. Optical depth resolution is approximately $7 \mu \mathrm{m}$ with digital resolution reaching $3.5 \mu \mathrm{m}$. Imaging was performed using the proprietary software package Eye Explorer (version 3.2.1.0, Heidelberg Engineering) and for the work-up of the images we used CorelX3 (Corel Corporation, Ottawa, ON, Canada). 
Retinal explant cultures. Organotypic retinal cultures were prepared as described previously. ${ }^{22,35}$ P5 animals were killed, their eyes enucleated, and incubated for $15 \mathrm{~min}$ with $0.12 \%$ proteinase $\mathrm{K}$ (ICN Biomedicals Inc., OH, USA; 193504). The activity of proteinase $\mathrm{K}$ was blocked by $10 \%$ fetal calf serum and rinsing with serum free medium. Cornea, sclera, lens, and choroid were removed, after which the retina, together with the RPE, was cut into a propeller-like shape with four wedges and transferred to a culture membrane insert (Millipore Carrigtwohill, Cork, Ireland; PIHA03050). The inserts were placed in six-well culture plates with R16 medium and supplements. ${ }^{22}$ Cultures were incubated at $37^{\circ} \mathrm{C}$ in a humidified $5 \% \mathrm{CO}_{2}$ incubator for 6 days, and the culture medium was changed every 2 days. For the first 2 days, the cultures were left without treatment and then treated for 4 days with $100 \mu \mathrm{M}$ Zaprinast (Sigma-Aldrich, Munich, Germany; Z0878) dissolved in dimethyl sulfoxide (DMSO; Sigma-Aldrich, D2650) and diluted in R16 medium with supplements. Only DMSO was added for the control groups.

IF and TUNEL assay. Animals were killed with $\mathrm{CO}_{2}$ and their eyes were enucleated. Eyecups were immersion fixed for $1 \mathrm{~h}$ at room temperature (RT) in $4 \%$ paraformaldehyde (PFA; Polysciences, Warrington, PA, USA) in $0.1 \mathrm{M}$ phosphate buffer ( $\mathrm{PB}, \mathrm{pH}$ 7.4) containing $0.2 \mathrm{M}$ sucrose. After washing in $\mathrm{PB}$, eyes were cryoprotected by immersion in graded sucrose $(10 \%, 20 \%, 30 \%)$ in PB. Tissues were then embedded in a tissue-freezing medium (Jung, Leica Instruments, Heidelberg, Germany). Vertical sections (12 $\mu \mathrm{m}$ ) were cut on a Leica CM3050S Microtome (Leica Biosystems, Wetzlar, Germany), air dried at $37^{\circ} \mathrm{C}$ for $1 \mathrm{~h}$, and stored at $-20^{\circ} \mathrm{C}$ until use. Frozen sections from fixed tissue were air dried for $30-60 \mathrm{~min}$ at $37^{\circ} \mathrm{C}$.

For the IF studies sections were rinsed in PBS and preincubated for $1 \mathrm{~h}$ at RT in blocking solution containing $10 \%$ normal goat serum, $1 \%$ bovine serum albumin, and $0.1 \%$ Triton X in PBS. Primary antibodies (see Table 1) were diluted in blocking solution overnight at $4{ }^{\circ} \mathrm{C}$. Subsequently, sections were rinsed in PBS and incubated with Alexafluor-488 conjugated secondary antibody (Invitrogen, Darmstadt, Germany; dilution 1:250-1:750). Sections were washed in PBS and mounted in Vectashield mounting medium with DAPI (Vector, Burlingame, CA, USA).

Cell death was assessed using the terminal deoxynucleotidyl transferase dUTP nick end labeling (TUNEL) assay by means of an in situ cell death detection kit with fluorescein isothiocyanate as the reporter fluorochrome (Roche Diagnostics, Mannheim, Germany).

PAR immunohistochemistry. PAR immunohistochemistry was performed with sections from in vivo and in vitro PARP1 KO, PARG110 KO, and wt. Sections were air dried for $30-60 \mathrm{~min}$ at $37^{\circ} \mathrm{C}$ and washed with PBS for $10 \mathrm{~min}$. Non-specific background reduced by quenching solution which included $30 \% \mathrm{H}_{2} \mathrm{O}_{2}, \mathrm{MeOH}, 0.1 \%$ PBST. After that, sections were blocked with $10 \%$ normal goat serum in $0.1 \%$ PBST for $1 \mathrm{~h}$ at $\mathrm{RT}$ and incubated with PAR antibody (PAR 10 H, Alexis, dilution $1: 200$ ) for overnight at $+4{ }^{\circ} \mathrm{C}$. Biotinylated secondary antibody (Vector lab; dilution 1:150) was diluted in $5 \%$ normal goat serum in $0.1 \%$ PBST and the sections were incubated for $1 \mathrm{~h}$ at RT. After washing, the slides were incubated in Vectastain Elite ABC kit (Vector lab) for $1 \mathrm{~h}$ at RT. The color reaction was produced with 3,3'diaminobenzidine (DAB) solution containing $20 \%$ Glucose, $0.4 \% \mathrm{NH}_{4} \mathrm{Cl}, 1 \%$ Nickel ammonium sulfate, $40 \mathrm{mg}$ DAB, $40 \mu \mathrm{l} \mathrm{Glucoseoxidase.} \mathrm{After} \mathrm{DAB} \mathrm{incubation,} \mathrm{slides}$ were washed with PB and covered by Aquatex (Merck, Darmstadt, Deutschland).

Histological staining. Morphologic ex vivo characterization of PARG110 KO retinae was performed after haematoxylin/eosin staining. Cryosectioned retinae, fixed with $4 \%$ PFA for $1 \mathrm{~h}$, were stained in Harris haematoxylin solution (Vector Lab, H-3401) for $3 \mathrm{~min}$. Retinal sections were washed in double distilled water for $1 \mathrm{~min}$, exposed for $2 \mathrm{~s}$ to $25 \%$ hydrochloric acid in ethanol, and counterstained in Accustain eosin Y solution (Sigma-Aldrich, HT-110-1-16) for $30 \mathrm{~s}$ to $1 \mathrm{~min}$. A 70 $-96-100 \%$ alcohol series was used for dehydration and followed by washing in xylene for $2 \mathrm{~min}$.

Table 1 Primary antibodies used in this study and their dilutions

\begin{tabular}{llc}
\hline Antigen & Source/Cat. number & Dilution \\
\hline Cleaved caspase-3 (Asp175) (5A1E) & Cell Signalling/9664 & $1: 300$ \\
Calbindin (D-28k) & Swant/300 & $1: 500$ \\
PKC $\alpha$ & Novus Biologicals/NB200 586 & $1: 50$ \\
PAR (10H) & Enzo/ALX-804-220 & $1: 200$ \\
PARG (PARG110/56) & Abcam/ab16060 & $1: 100$ \\
\hline
\end{tabular}

Activity assays for calpain and PARP. Eyes from PARG110 KO, PARP1 $\mathrm{KO}$, wt, and $r d 1$ mice were enucleated without fixation, frozen immediately on dry ice $\left(-72^{\circ} \mathrm{C}\right)$, and cryosectioned. Calpain activity was performed on unfixed retinal sections by first incubating them for $15 \mathrm{~min}$ in calpain reaction buffer (CRB: $25 \mathrm{mM}$ HEPES, $65 \mathrm{mM} \mathrm{KCl}, 2 \mathrm{mM} \mathrm{MgCl}, 1.5 \mathrm{mM} \mathrm{CaCl}_{2}$ ). Later, the fluorogenic compound CMAC, t-BOC-Leu-Met; 7-amino-4-chloromethylcoumarin, t-BOC-Lleucyl-L-methionine amide $(10 \mu \mathrm{M})$ was added to CRB together with $2 \mathrm{mM}$ DTT and the slides were incubated for $2 \mathrm{~h}$ in the dark. Slides were then washed in CRB two times, $10 \mathrm{~min}$ and mounted with Vectashield (Vector) before microscopy.

For PARP activity, unfixed tissue sections were incubated with an avidin-biotin blocking kit (Vector) according to the manufacturer's instructions to block endogenous biotin and to reduce background. The slides were then washed twice for $5 \mathrm{~min}$ in PBS and incubated with PARP reaction mixture $(10 \mathrm{mM} \mathrm{MgCl}, 1 \mathrm{mM}$ dithiothreitol, $5 \mu \mathrm{M}$ biotinylated NAD (Trevigen, Gaithersburg, MD, USA) in $100 \mathrm{mM}$ Tris buffer with $0.2 \%$ Triton $\mathrm{X} 100, \mathrm{pH} 8.0$ ) for $2.5 \mathrm{~h}$ at $37^{\circ} \mathrm{C}$. Then the sections were washed with PBS, three times for $5 \mathrm{~min}$. The biotin incorporated by PARP activity was then detected by fluorescently labeled avidin (1:800 in PBS, $1 \mathrm{~h}$ at RT). After three times $5 \mathrm{~min}$ washing in PBS, the sections were mounted in Vectashield (Vector). For controls, biotinylated $\mathrm{NAD}^{+}$was omitted from the reaction mixture resulting in absence of detectable reaction product.

HDAC activity assay. The HDAC activity assay was performed on cryosections from 4\% PFA fixed eyes. The assay is based on an adaptation of the Fluor de Lys Fluorescent Assay System (Biomol, Hamburg, Germany). Retinal sections were incubated for $3 \mathrm{~h}$ at RT with $200 \mu \mathrm{M}$ Fluor de Lys-SIRT2 deacetylase substrate (Biomol) in assay buffer $(50 \mathrm{mM}$ Tris/ $\mathrm{HCl}, \mathrm{pH}$ 8.0; $137 \mathrm{mM}$ $\mathrm{NaCl} ; 2.7 \mathrm{mM} \mathrm{KCl} ; 1 \mathrm{mM} \mathrm{MgCl} 2$ ). The sections were washed once for $5 \mathrm{~min}$ in PBS and then fixed again for $20 \mathrm{~min}$ in $100 \%$ methanol at $-20{ }^{\circ} \mathrm{C}$. The slides were incubated in Developer II (Biomol) solution, diluted 1:10 in assay buffer, and immediately coversliped and photographed under the microscope. ${ }^{22}$

Microscopy, cell counting and statistics. Microscopy was performed by a Zeiss (Göttingen, Deutschland) Imager Z1 Apotome Microscope. Images were taken with a Zeiss Axiocam digital camera using the Zeiss Axiovision 4.7 software. Corel Draw X3 software was used for image enhancements (Contrast, Colors).

The percentages of positive cells in the different assays (PARP activity, PAR IF, Calpain activity, Caspase-3 IF, HDAC activity, TUNEL) were assessed and calculated in a blinded fashion as reported previously. ${ }^{5,23}$ For each animal, three fields of view at $\times 20$ magnification in central retinal areas (in proximity to the optic nerve) were analyzed and at least three sections were quantified. At least three different animals were analyzed for each time point and genotype.

Values are given as mean \pm S.E.M. Statistical analysis was performed using GraphPad Prism 4.01 software (GraphPad Software, La Jolla, CA, USA), two-tailed Student's t-test was used for single comparison and one-way ANOVA test with Bonferroni correction was used for multiple comparisons. Levels of significance were: ${ }^{\star} P<0.05,{ }^{\star \star} P<0.01,{ }^{* \star *} P<0.001$

\section{Conflict of Interest}

The authors declare no conflict of interest.

Acknowledgements. We thank Karina Gültig, Sandra Bernhard-Kurz, and Klaudija Masarini for excellent technical assistance. This work has been supported by grants from the Kerstan Foundation, the EU (NEUROTRAIN: MEST-CT-2005-020235), and Deutsche Forschungsgemeinschaft (DFG; PA1751/4-1, Se837/5-2, Se837/6-2).

1. Ferrari S, Di IE, Barbaro V, Ponzin D, Sorrentino FS, Parmeggiani F. Retinitis pigmentosa: genes and disease mechanisms. Curr Genomics 2011; 12: 238-249.

2. Sancho-Pelluz J, Arango-Gonzalez B, Kustermann S, Romero FJ, van Veen T, Zrenner E et al. Photoreceptor cell death mechanisms in inherited retinal degeneration. Mol Neurobio 2008; 38: 253-269.

3. Trifunovic D, Sahaboglu A, Kaur J, Mencl S, Zrenner E, Ueffing M et al. Neuroprotective strategies for the treatment of inherited photoreceptor degeneration. Curr Mol Med 2012; 12: 598-612.

4. Paquet-Durand F, Silva J, Talukdar T, Johnson LE, Azadi S, van Veen T et al. Excessive activation of poly(ADP-ribose) polymerase contributes to inherited photoreceptor degeneration in the retinal degeneration 1 mouse. J Neurosci 2007; 27: 10311-10319. 
5. Sahaboglu A, Tanimoto N, Kaur J, Sancho-Pelluz J, Huber G, Fahl E et al. PARP1 gene knock-out increases resistance to retinal degeneration without affecting retinal function. PLOS One 2010; 5: e15495.

6. Kaur J, Mencl S, Sahaboglu A, Farinelli P, van Veen T, Zrenner E et al. Calpain and PARP activation during photoreceptor cell death in $\mathrm{P} 23 \mathrm{H}$ and S334ter rhodopsin mutant rats. PLoS One 2011; 6: e22181.

7. Dantzer F, Ame JC, Schreiber V, Nakamura J, Menissier-de MJ, de MG. Poly(ADP-ribose) polymerase-1 activation during DNA damage and repair. Methods Enzymol 2006; 409 : 493-510.

8. Hassa PO, Hottiger MO. The diverse biological roles of mammalian PARPS, a small but powerful family of poly-ADP-ribose polymerases. Front Biosci 2008; 13: 3046-3082.

9. Virag L, Szabo C. The therapeutic potential of poly(ADP-ribose) polymerase inhibitors. Pharmacol Rev 2002; 54: 375-429.

10. Bowes C, Li T, Danciger M, Baxter LC, Applebury ML, Farber DB. Retinal degeneration in the rd mouse is caused by a defect in the beta subunit of rod cGMP-phosphodiesterase. Nature 1990; 347: 677-680.

11. Zhang $\mathrm{X}$, Feng $\mathrm{Q}$, Cote $\mathrm{RH}$. Efficacy and selectivity of phosphodiesterase-targeted drugs in inhibiting photoreceptor phosphodiesterase (PDE6) in retinal photoreceptors. Invest Ophthalmol Vis Sci 2005; 46: 3060-3066.

12. Sahaboglu A, Paquet-Durand O, Dietter J, Dengler K, Bernhard-Kurz S, Ekstrom PA et al. Retinitis pigmentosa: rapid neurodegeneration is governed by slow cell death mechanisms. Cell Death Dis 2013; 4: e488.

13. Du J, Cleghorn WM, Contreras L, Lindsay K, Rountree AM, Chertov AO et al. Inhibition of mitochondrial pyruvate transport by Zaprinast causes massive accumulation of aspartate at the expense of glutamate in the retina. J Biol Chem 2013; 288: 36129-36140.

14. Zahradka P, Ebisuzaki K. A shuttle mechanism for DNA-protein interactions. The regulation of poly(ADP-ribose) polymerase. Eur J Biochem 1982; 127: 579-585.

15. D'Amours D, Desnoyers S, D'Silva I, Poirier GG. Poly(ADP-ribosyl)ation reactions in the regulation of nuclear functions. Biochem J 1999; 342: 249-268.

16. Putt KS, Hergenrother PJ. A nonradiometric, high-throughput assay for poly(ADP-ribose) glycohydrolase (PARG): application to inhibitor identification and evaluation. Anal Biochem 2004; 333: 256-264.

17. Burns DM, Ying W, Kauppinen TM, Zhu K, Swanson RA. Selective down-regulation of nuclear poly(ADP-ribose) glycohydrolase. PLoS One 2009; 4: e4896.

18. Mortusewicz O, Fouquerel $\mathrm{E}$, Ame JC, Leonhardt $\mathrm{H}$, Schreiber V. PARG is recruited to DNA damage sites through poly(ADP-ribose)- and PCNA-dependent mechanisms. Nucleic Acids Res 2011; 39: 5045-5056.

19. Cortes U, Tong WM, Coyle DL, Meyer-Ficca ML, Meyer RG, Petrilli V et al. Depletion of the 110-kilodalton isoform of poly(ADP-ribose) glycohydrolase increases sensitivity to genotoxic and endotoxic stress in mice. Mol Cell Biol 2004; 24: 7163-7178.

20. Arango-Gonzalez B, Cellerino A, Kohler K. Exogenous brain-derived neurotrophic factor (BDNF) reverts phenotypic changes in the retinas of transgenic mice lacking the BDNF gene. Invest Ophthalmol Vis Sci 2009; 50: 1416-1422.

21. Martinez-Zamudio R, Ha HC. Histone ADP-ribosylation facilitates gene transcription by directly remodeling nucleosomes. Mol Cell Biol 2012; 32: 2490-2502.

22. Sancho-Pelluz J, Alavi M, Sahaboglu A, Kustermann S, Farinelli P, Azadi S et al. Excessive HDAC activation is critical for neurodegeneration in the rd1 mouse. Cell Death Dis 2010 1: $1-9$
23. Paquet-Durand F, Hauck SM, van Veen T, Ueffing M, Ekstrom P. PKG activity causes photoreceptor cell death in two retinitis pigmentosa models. J Neurochem 2009; 108: $796-810$.

24. Shimokawa T, Masutani M, Ishiguro $\mathrm{Y}$, Araki S, Aoki $\mathrm{Y}$, Nakagama $\mathrm{H}$ et al. Linkage mapping of the rat poly(ADP-ribose) glycohydrolase (Parg) gene to chromosome 16 Exp Anim 1999; 48: 217-218.

25. Wang Y, Dawson VL, Dawson TM. Poly(ADP-ribose) signals to mitochondrial AIF: a key event in parthanatos. Exp Neurol 2009; 218: 193-202.

26. Satoh MS, Lindahl T. Role of poly(ADP-ribose) formation in DNA repair. Nature 1992; 356 356-358.

27. Sims JL, Berger SJ, Berger NA. Poly(ADP-ribose) Polymerase inhibitors preserve nicotinamide adenine dinucleotide and adenosine 5'-triphosphate pools in DNA-damaged cells: mechanism of stimulation of unscheduled DNA synthesis. Biochemistry 1983; 22: 5188-5194.

28. Buonvicino D, Formentini L, Cipriani G, Chiarugi A. Glucose deprivation converts poly(ADP-ribose) polymerase-1 hyperactivation into a transient energy-producing process J Biol Chem 2013; 288: 36530-36537.

29. Kahlert S, Reiser G. Glial perspectives of metabolic states during cerebral hypoxiacalcium regulation and metabolic energy. Cell Calcium 2004; 36: 295-302.

30. Finch KE, Knezevic CE, Nottbohm AC, Partlow KC, Hergenrother PJ. Selective smal molecule inhibition of poly(ADP-ribose) glycohydrolase (PARG). ACS Chem Biol 2012; 7 563-570.

31. Blenn C, Althaus FR, Malanga M. Poly(ADP-ribose) glycohydrolase silencing protects against H2O2-induced cell death. Biochem J 2006; 396: 419-429.

32. Tanimoto N, Muehlfriedel RL, Fischer MD, Fahl E, Humphries P, Biel M et al. Vision tests in the mouse: functional phenotyping with electroretinography. Front Biosci 2009; 14 $2730-2737$.

33. Tanimoto N, Sothilingam V, Seeliger MW. Functional phenotyping of mouse models with ERG. Methods Mol Biol 2013; 935: 69-78.

34. Fischer AJ, Scott MA, Tuten W. Mitogen-activated protein kinase-signaling stimulates Muller glia to proliferate in acutely damaged chicken retina. Glia 2009; 57: 166-181.

35. Caffe AR, Soderpalm AK, Holmqvist I, van Veen T. A combination of CNTF and BDNF rescues rd photoreceptors but changes rod differentiation in the presence of RPE in retinal explants. Invest Ophthalmol Vis Sci 2001; 42: 275-282.

(c) (2) Cell Death and Disease is an open-access journa BY licensed under a Creative Commons Attribution-NonCommercialShareAlike 3.0 Unported License. The images or other third party material in this article are included in the article's Creative Commons license, unless indicated otherwise in the credit line; if the material is not included under the Creative Commons license, users will need to obtain permission from the license holder to reproduce the material. To view a copy of this license, visit http://creativecommons.org/licenses/ by-nc-sa/3.0/

Supplementary Information accompanies this paper on Cell Death and Disease website (http://www.nature.com/cddis) 